\title{
Influence de la compétition et de la disponibilité en eau sur l'importance de l'aubier du douglas
}

\author{
H. POLGE \\ I.N.R.A., Station de Recherches sur la Qualité des Bois, \\ Centre de Recherches forestières de Nancy, \\ Champenoux, F 54280 Seichamps
}

\begin{abstract}
Résumé
Les 334 arbres étudiés sont des douglas de 11 provenances différentes âgés de 13 ans dont une moitié a été irriguée en 1976 et 1977 et l'autre non. L'aubier a été délimité par 2 méthodes : sur la base de sa teneur en eau supérieure (par comparaison des radiographies à l'état frais et à l'état sec de carottes de sondage de $5 \mathrm{~mm}$ ) et par sa différence de couleur, observée sur la carotte sèche. Deux modalités d'expression ont été utilisées : la largeur et le nombre d'années.

Un décalage de presque un an et de $5 \mathrm{~mm}$ dans le sens radial existe entre la méthode couleur et la méthode humidité, la duraminisation intervenant postérieurement au départ de l'eau.

Aucun effet provenance n'a été observé, mais il existe un très fort effet ligne dû aux 2 lisières où l'aubier est plus large, mais s'étend sur un plus petit nombre d'années. Ce nombre est très significativement plus élevé dans les arbres irrigués que dans les témoins et la largeur y est supérieure.

Les corrélations entre les deux méthodes sont très étroites, mais celles qui ont été calculées entre les deux modes d'expression ne sont pas significatives; la raison en est que nombres et largeurs varient en sens contraire chez les dominants et dans le même sens chez les dominés.

De très nombreuses zones sèches ont été dénombrées dans l'aubier; les plus fréquentes ont une largeur supérieure à $0,5 \mathrm{~mm}$ dans le sens radial et se trouvent dans le bois initial. Il est difficile d'expliquer leur présence par des facteurs climatiques simples, mais une liaison négative très significative a été mise en évidence entre la fréquence de ces zones sèches et la densité minimale annuelle du bois.
\end{abstract}

\section{Introduction}

\subsection{Importance technologique de la duraminisation}

La transformation de laubier en bois de cœur entraîne des modifications chimiques (dépôt de substances phénoliques, disparition de l'amidon) et physiques (formation de 
thylles dans les vaisseaux des angiospermes et blocage des ponctuations aréolées chez les gymnospermes) qui conditionnent souvent leurs possibilités d'emploi :

Le cas le plus net est celui des feuillus durs pour lesquels la norme française NF 53-501 exclut du classement en avivés de choix exceptionnel ou de $1^{\text {er }}$ choix toute pièce comportant de l'aubier, et ne tolère la présence d'aubier que sur une face pour le classement en $2^{\circ}$ et $3^{\circ}$ choix. Ces spécifications s'expliquent par l'altérabilité de la partie vivante de larbre (en raison de la présence de matières nutritives de réserve et des facilités de communication entre cellules) et, à l'inverse, par la relative résistance du duramen aux attaques des divers agents biologiques de dégradation, que lui confèrent l'absence de matériaux glucidiques assimilables, l'imprégnation de ses cellules par des substances aromatiques dont certaines exercent une action antifongique et l'existence d'obstacles physiques pour le passage entre éléments anatomiques ou à l'intérieur de ceux-ci.

Cependant, la présence d'un aubier important ne constitue pas toujours un défaut : elle est au contraire recherchée pour les bois de papeterie d'une part en raison de sa plus grande accessibilité aux liqueurs de cuisson, d'autre part du fait des dépenses accrues de blanchiment qu'entraine souvent la forte coloration du bois de cxur.

Par ailleurs, si l'aubier est plus altérable, il est aussi, par chance, plus facile à traiter par des produits de préservation : c'est ainsi que pour l'essence qui nous intéresse ici, le douglas, la norme canadienne A 56-1942 concernant les utilisations en pilots impose une largeur minimale d'aubier de $20 \mathrm{~mm}$ pour les bois soumis à un traitement antiseptique (pour les bois non traités, au contraire, est fixée une limite naximale à ne pas dépasser : 20 p. 100 sur le diamètre au gros bout).

Les problèmes de circulation des fluides dans laubier ne sont cependant pas toujours simples à lésoudre, et certaines anomalies restent difficiles à expliquer ; une des plus notables concerne le genre épicéa où certaines espèces de l'Est canadien (Picea glauca, Picea mariana, Picea rubens) ont un aubier relativement accessible aux produits de préservation, alors que notre épicéa commun (Picea abies) est au contraire réfractaire à tout traitement.

\subsection{Caractères distinctifs de l'aubier et du bois de cour}

Un certain nombre, de nature chimique ou physique, mais en rapport pour ceux-ci avec l'anatomie du bois, ont déjà été mentionnés au paragraphe précédent; ce ne sont pas les seuls, et, par ailleurs, ils ne présentent pas un caractère de généralité absolue : ainsi, l'absence d'amidon dans les cellules de parenchyme ne suffit pas pour prouver qu'il y a duraminisation, car sa teneur à l'intérieur même de l'aubier varie considérablement en fonction de l'activité physiologique de l'arbre (BAMBER \& Humphreys, 1965), et il peut disparaître complètement avant la limite du bois de cour. La cessation de toute activité biologique est, elle aussi, souvent considérée comme liée au passage au duramen; il a cependant été démontré (Frey-WYssling $\&$ BosshaRd, 1959) que les noyaux cellulaires deviennent plus petits, puis disparaissent, et que les mitochondries peuvent ne plus être fonctionnelles bien avant cette limite.

Certains critères ne caractérisent la duraminisation qu'au niveau de l'espèce ou du groupe d'espèces : ainsi les cellules des canaux résinifères radiaux s'épaissis- 
sent-elles par formation d'une membrane secondaire dans le bois de cæur des pins de la section Haploxylon, mais non dans ceux de la section Diploxylon (BAMBER, 1970 et BAMBER, 1972).

Un des caractères les plus importants de séparation de l'aubier et du bois de cœur est le taux d'humidité du bois, lui-même lié aux possibilités de transport d'eau et de sève brute dans la partie vivante de l'arbre. Mais, là aussi, la réalité est complexe : comme l'a montré Stewart (1967), il existe, à l'intérieur de l'aubier, des variations notables résultant, d'une part, des fluctuations en fonction des besoins de la transpiration, du flux qui transite par les couches les plus externes du bois, d'autre part, de la présence, dans les zones moyennes et internes de ce même aubier, d'un stock d'eau variable pouvant, en cas de besoin, alimenter le courant ascendant via le parenchyme des rayons.

En règle générale, la teneur en eau de l'aubier varie en raison inverse cie sa vitesse d'ascension qui, elle-même augmente avec la longueur des éléments conducteurs libre d'obstacle, c'est-à-dire avec la longueur entre deux cloisons successives dans le sens vertical. Ainsi, les vitesses diminuent de 50 à 5 puis à $0,5 \mathrm{~m} / \mathrm{h}$ en moyenne en passant des feuillus à zone poreuse aux feuillus à pores diffus, puis aux résineux, et, corrélativement, le taux d'humidité de leur aubier augmente depuis des valeurs inférieures à 75 p. 100 pour les premiers à des valeurs voisines de 100 p. 100 pour les seconds et supérieures à 130 p. 100 pour les derniers.

Une délimitation aubier-bois de cæur basée sur leur différence de teneur en eau sera donc relativement facile à obtenir chez les gymnospermes, malgré la présence occasionnelle dans le premier de zones sèches plus ou moins étroites et plus ou moins marquées (Polge, 1964). Le cas des angiospermes est assez différent, puisqu'il y arrive même, comme chez les caryas, que ce soit le bois de cœur qui présente le taux d'humidité le plus élevé.

\subsection{Déterminisme physiologique de la duraminisation}

La transformation de l'aubier en bois de cœur a longtemps été considérée comme résultant d'un phénomène naturel de vieillissement, puis de mortalité sous l'effet d'une accumulation de polyphénols atteignant un seuil de toxicité (RUdman, 1966), d'une accumulation d'air dans un tissu cellulaire devenu clos (Priestley, 1932) ou de la dévitalisation du parenchyme par des micro-organismes (Margaret CHATTAWAY, 1952).

Cependant BAmBer (1975) a émis l'hypothèse, étayée par un certain nombre d'éléments de preuve, que la mort des cellules de parenchyme est la conséquence, et non la cause, de la formation du bois de cæur. Celle-ci résulterait d'un processus de croissance secondaire, analogue à la chute des feuilles ou à la maturation des fruits, déclenché par une substance hormonale circulant de façon centripète dans les rayons et atteignant son seuil d'efficacité à la limite du bois de cour, où ne pouvant plus avancer, elle s'accumulerait puis diffuserait dans un plan tangentiel. Pour justifier sa façon de voir, il note que certains processus physiologiques sont relativement intenses dans la zone de transition comme la respiration ou même le développement cellulaire, avec la formation des thylles ou parfois l'épaississement 
des membranes (canaux résinifères radiaux ou rayons médullaires). Observant qu'il existe le plus souvent des corrélations étroites entre le diamètre des arbres et la largeur de leur aubier, il en déduit que la duraminisation est un processus régulateur destiné à limiter la surface relative de celui-ci au strict niveau nécessaire à la conduction de la sève et à l'approvisionnement des cellules vivantes en matières minérales et en hydrates de carbone, la transformation en bois de cour des couches internes devenues inutiles dans cette optique compensant l'apparition de nouvelles couches du fait de la croissance cambiale.

Le but du présent travail n'est pas d'approfondir les mécanismes physiologiques en cause dans la duraminisation du bois; il a seulement été entrepris pour tirer parti d'un dispositif expérimental, certes très peu satisfaisant du point de vue de l'analyse statistique, mais à partir duquel il paraissait possible d'étudier les effets, sur la formation du bois de cœur, de la disponibilité en eau des arbres, de leur vigueur et de leur hérédité au niveau infraspécifique.

\section{Matériel et méthodes}

\subsection{Dispositif expérimental}

Les observations dont il est rendu compte ici ont été faites sur des douglas verts [Pseudotsuga menziesii (Mirb.) Franco] de 11 provenances différentes (10 d'origine américaine et une provenance artificielle française), installés au printemps 1965 dans l'agrandissement de l'Arboretum d'Amance (Meurthe-et-Moselle). Chacune d'elles y est représentée par un placeau de 10 lignes, à raison de 4 arbres par ligne, à une équidistance de $1,80 \mathrm{~m}$, sans répétition. Le plan du dispositif est donné en figure 1 .

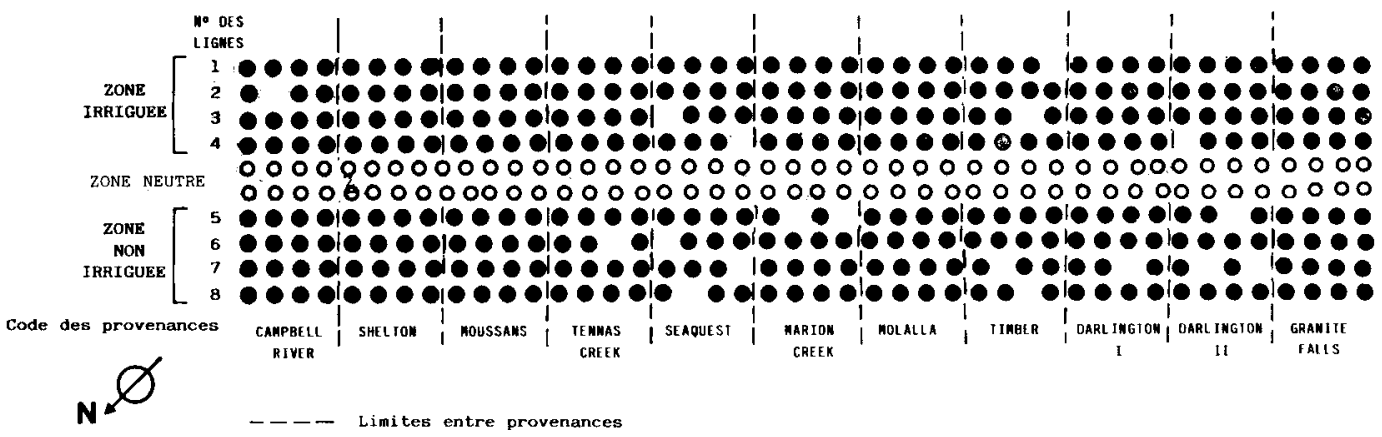

FIG. 1

Plan du dispositif.

Zone irriguée : irrigated area.

Experimental design.

Neutre : neutral.

Code des provenances : code name of provenances.

$\mathrm{N}^{0}$ des lignes : number of the lines.

Limite entre provenances : Provenance boundaries. 
En 1976, ce dispositif a été utilisé pour étudier l'influence de l'alimentation en eau sur la croissance des arbres (Aussenac, 1980), en comparant des arbres soumis à la sécheresse sévère qui sévit cette année-là à d'autres ayant bénéficié d'une irrigation compensatrice à l'aide d'un système goutte-à-goutte. En pratique, les arbres des lignes 1 à 4 ont reçu en 1976 en apport artificiel léquivalent de $414 \mathrm{~mm}$ de pluie, et en 1977, l'équivalent de $172 \mathrm{~mm}$. Une ration de survie de $25 \mathrm{~mm}$ a été délivrée aux témoins des lignes 5 à 8 en 1976, les deux lignes intermédiaires étant considérées comme neutres.

\subsection{Echantillonnage}

Tous les individus encore vivants en 1978 ont été étudiés; ils représentaient un total de 334 arbres sur 352, les 18 manquants se répartissant entre un minimum de zéro et un maximum de 5 par provenance, entre un minimum de 1 et un maximum de 5 par ligne, et affectant à raison de 7 la partie irriguée et de 11 la zone témoin. Les arbres ont tous été sondés à hauteur d'homme avec une tarière de $5 \mathrm{~mm}$ introduite du côté ouest des tiges.

\subsection{Mesures}

Aussitôt prélevées, les carottes ont été placées dans des tubes de verre de diamètre approprié, et transférées au laboratoire où elles ont été radiographiées suivant une méthodologie déjà décrite (Pol.ge, 1966), en utilisant les conditions d'exposition suivantes :

- distance source-film : $2,5 \mathrm{~m}$;

— intensité du flux électronique : 20 milliampères;

- tension appliquée aux bornes du tube : $15 \mathrm{kV}$;

- durée d'exposition : $4 \mathrm{mn} 30 \mathrm{~s}$.

Elles ont ensuite été conditionnées à 10 p. 100 d'humidité dans une étuve hygrométrique, puis radiographiées à nouveau dans les mêmes conditions que précédemment.

L'ensemble des 668 images radiographiques obtenues a alors fait l'objet de tirages positifs au grossissement de 2,06, et la comparaison des 2 clichés successifs d'une même carotte préalablement juxtaposés a donné une première délimitation de l'aubier basée sur sa teneur en eall supérieure (Por.ge, 1964).

On a mesuré sur le positif à l'état sec les paramètres suivants :

- largeur de l'aubier (méthode de l'humidité) : LAH ;

- nombre d'années d'aubier (méthode de l'humidité) : NAH ;

- largeur de la tranche de cernes 1972-1975 inclusivement : L 1 ;

- largeur de la tranche de cernes 1976-1978 : L 2.

Enfin, sur les carottes à l'état sec, on a procédé à une autre délimitation de l'aubier basée sur la différence de couleur (coloration rosée caractéristique du bois de douglas apparaissant au moment de la duraminisation). Le repérage précis de 
cette limite est apparu généralement facile; en cas de doute, une vérification a été faite sur la génératrice opposée de la carotte, et les quelques échantillons pour lesquels une difficulté subsistait ont été débités sous forme de sections transversales de $1,6 \mathrm{~mm}$ d'épaisseur à laaide de scies-fraises jumelées, ce qui donne des surfaces extrêmement lisses où les changements de couleur du bois sont très apparents. On a ainsi pu mesurer 2 autres paramètres :

- largeur de laubier (méthode de la couleur) : LAC;

- nombre d’années d’aubier (méthode de la couleur) : NAC.

En ce qui concerne NAH et NAC, il s'agit de nombres-code attribués pour chaque année à chacune des 4 zones suivantes : à l'intérieur du bois initial (BI), à la limite bois initial - bois final (BI-BF), à l'intérieur du bois final (BF), sur la limite avec l'année suivante, d’où le tableau d’équivalence ci-dessous :

\begin{tabular}{|c|c|c|c|}
\hline $\begin{array}{l}\text { Nombre } \\
\text { code }\end{array}$ & $\begin{array}{l}\text { Nombre } \\
\text { code }\end{array}$ & $\begin{array}{c}\text { Nombre } \\
\text { code }\end{array}$ & $\begin{array}{l}\text { Nombre } \\
\text { code }\end{array}$ \\
\hline $1=$ limite $75-76$ & $2=\mathrm{BF} 75$ & $3=\mathrm{BI}-\mathrm{BF} 75$ & $4=\mathrm{BI} 75$ \\
\hline $5=$ limite $74-75$ & $6=\mathrm{BF} 74$ & $7=\mathrm{BI}-\mathrm{BF} 74$ & $8=\mathrm{BI} 74$ \\
\hline $9=$ limite $73-74$ & $10=\mathrm{BF} 73$ & $11=\mathrm{BI}-\mathrm{BF} 73$ & $12=\mathrm{BI} 73$ \\
\hline $13=$ limite $72-73$ & $14=\mathrm{BF} 72$ & $15=\mathrm{BI}-\mathrm{BF} 72$ & $16=\mathrm{BI} 72$ \\
\hline $17=$ limite $71-72$ & $18=\mathrm{BF} 71$ & $19=\mathrm{BI}-\mathrm{BF} 71$ & $20=\mathrm{BI} 71$ \\
\hline $21=$ limite $70-71$ & $22=\mathrm{BF} 70$ & $23=\mathrm{BI}-\mathrm{BF} 70$ & $24=\mathrm{BI} 70$ \\
\hline $25=$ limite $69-70$ & $26=\mathrm{BF} 69$ & $27=\mathrm{BI}-\mathrm{BF} 69$ & \\
\hline
\end{tabular}

\section{Résultats}

\subsection{Moyennes générales}

Les valeurs trouvées sont les suivantes (toutes les largeurs sont exprimées en grandeurs vraies à l'échelle 1/1) :

- NAH : 15,36 (limite vers la fin du bois initial 1972);

- NAC : 18,43 (limite vers le début du bois final 1971);

- LAH : $26,3 \mathrm{~mm}$;

- LAC : $31,1 \mathrm{~mm}$;

- L 1 : 19,05 mm, soit une moyenne annuelle de 4,76 mm;

- L 2 : 9,25 mm, soit une moyenne annuelle de $3,08 \mathrm{~mm}$.

Un décalage sensible apparaît entre les deux méthodes de délimitation de laubier : la coloration du duramen se produit presque un an après que le bois soit devenu physiologiquement $\mathrm{sec}$, et corrélativement, la mesure basée sur l'humidité conduit à une largeur d'aubier inférieure d'environ $5 \mathrm{~mm}$.

En fïgure 2 est donné le diagramme de répartition des limites aubier-duramen pour les 2 méthodes; mise à part la confirmation de la différence mentionnée ci-dessus, on y observe une prépondérance très nette des individus pour lesquels l'apparition du bois de cœur coïncide avec un changement d'année ; cela ne surprend 


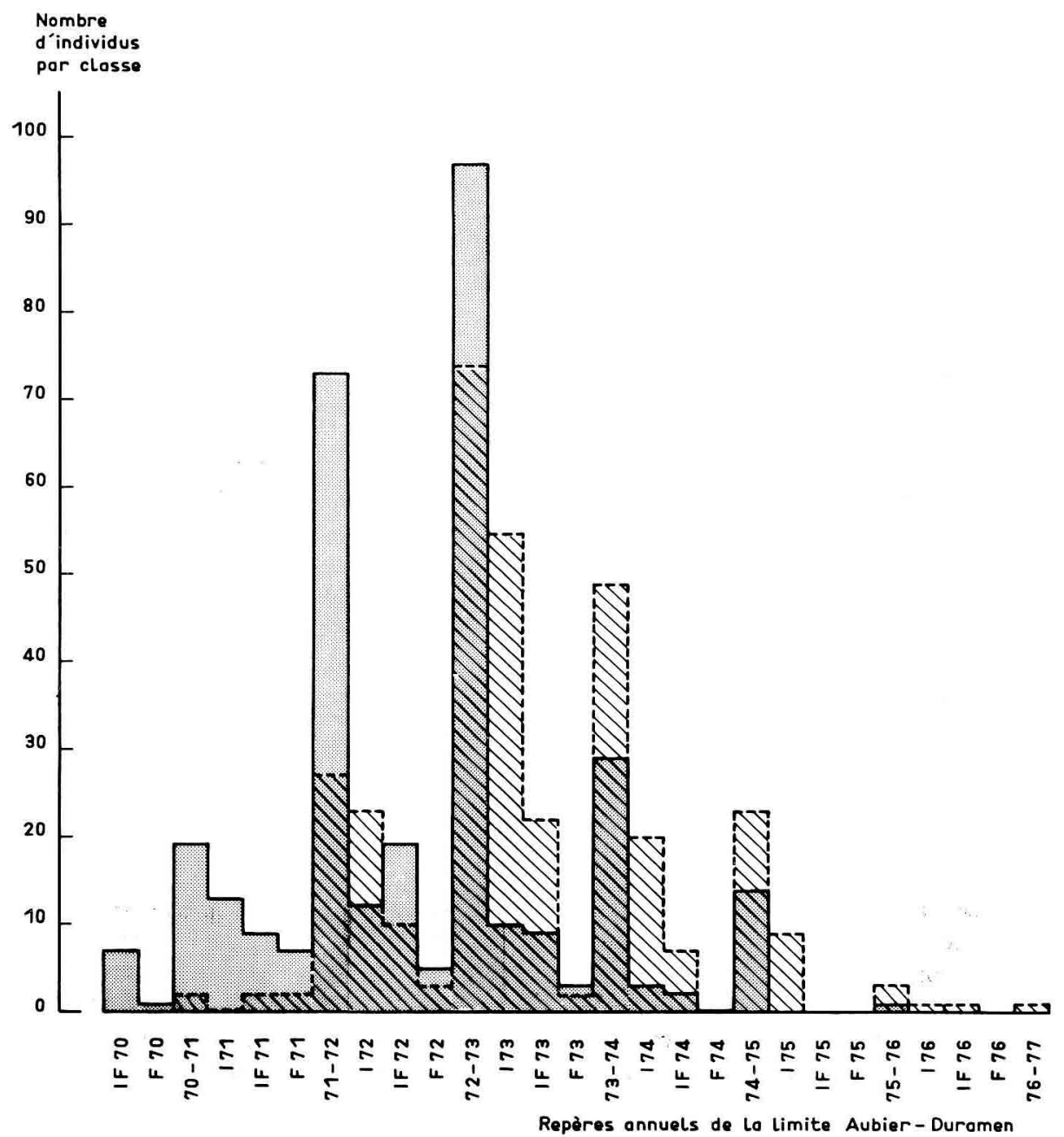

Delimitotion de L'Aubier et du Duromen:

$\triangle 8$ par la différence de teneur en eau du bois

por lo différence de couleur du bois

FIG. 2

Histogramme des limites aubier-duramen. Distribution of the limits between sapwood and heartwood.

Nombre d'individus par classe : number of trees per class.

Repères annuels : annual indicators ( $F$ : latewood; $I$ : early wood; $I F$ : limit between earlywood and latewood).

Délimitation de l'aubier et du duramen : delimitation between sapwood and heartwood. Par la différence de teneur en eau : according to the difference in moisture content. Par la différence de couleur du bois : according to the difference in color. 
guère car le passage du bois final d'une année au bois initial de l'année suivante s'accompagne de modifications très importantes au niveau cellulaire et infracellulaire, qui peuvent entraîner un brusque changement de vitesse pour les phénomènes physiques, chimiques et biochimiques liés à la duraminisation. Il convient d'ailleurs de dire que la délimitation du bois de cœur basée sur sa différence de couleur devient de plus en plus difficile au fur et à mesure que l'on se rapproche de la limite de cerne, en raison de la coloration de plus en plus foncée du bois final; quelques coïncidences avec des changements d'années peuvent dès lors être dues à un artefact, mais, dans ce cas, il n’aurait pu conduire qu'à une sous-estimation de la différence relevée entre les deux méthodes.

Une autre observation doit être faite quant aux moyennes générales : elle concerne la nette tendance à la diminution des largeurs de cerne qui surprend chez des douglas âgés seulement de 13 ans à la fin de l'expérience; la réduction moyenne de l'ordre de 35 p. 100 qui apparaît pour la période 1976-1978 par rapport à la période 1972-1975 ne s'explique pas seulement par la sécheresse de 1976, car les arbres irrigués présentent le même gradient que les témoins, et aussi parce qu'un ralentissement de la croissance se manifestait déjà entre 1972 et 1975. La véritable origine du phénomène réside dans la vigueur de la compétition entre arbres dans un peuplement planté à forte densité (3086 tiges/ha) et jamais éclairci ; cela mérite d’être signalé, car la vivacité de la concurrence est sans doute également responsable de la précocité de la duraminisation dans ces placeaux.

\subsection{Analyses de variance}

A défaut d'une analyse hiérarchique, il a été procédé, pour chaque variable, à 2 analyses à 2 facteurs contrôlés, pour tester les effets ligne et provenance d'une part, et les effets traitement et provenance d'autre part. Pour tenir compte de la non-orthogonalité du dispositif, des ajustements ont été faits successivement pour les 2 sources de variation en cause dans chaque cas, mais, du fait d'une répartition pas trop déséquilibrée des arbres manquants, les résultats obtenus sont extrêmement voisins; ne seront donc donnés ci-après que ceux obtenus après ajustement pour les facteurs ligne et traitement qui, seuls, sont apparus comme susceptibles d'exercer une influence significative sur tout ou partie des variables.

\subsection{Effets ligne et provenance}

Les valeurs des tests « $F$ » obtenues sont regroupées dans le tableau ci-dessous :

\begin{tabular}{|c|c|c|}
\hline Variable & $« F »$ provenance & $\ll F »$ ligne \\
\hline NAH & 1,143 & $11,993 * * *$ \\
\hline $\mathrm{NAC}$ & 0,465 & $10,222 * * *$ \\
\hline$\ldots \ldots \ldots \ldots$ & 0,470 & $13,857 * * *$ \\
\hline LAC $\ldots \ldots \ldots \ldots \ldots$ & 1,044 & $14,498 * * *$ \\
\hline L $1 \ldots \ldots$ & 1,207 & $12,641 * * *$ \\
\hline L 2 . & 0,552 & $37,027 * * *$ \\
\hline
\end{tabular}


Les valeurs-limites aux seuils de signification de 5 p. 100,1 p. 100 et 0,1 p. 100 sont respectivement de $2,96,2,32$ et 1,83 pour l'effet provenance (10 et 316 D.L.) et de $3,47,2,64$ et 2,01 pour l'effet ligne (7 et 316 D.L.).

On note tout d'abord l'absence totale d'effet provenance: pour les critères de croissance (L 1 et L 2), elle peut s'expliquer par le fait que seules des origines susceptibles de pousser convenablement dans les conditions de l'Arboretum d'Amance sont représentées, et aussi, la diminution de la valeur de «F» de $\mathbf{L} 1$ à $\mathrm{L} 2$ le prouve, par l'influence égalisatrice de la compétition en absence d'éclaircie.

Si aucun des critères de quantification de l'aubier napparaît comme soumis à une variabilité inter-provenance, il serait prématuré d'en déduire que tout déterminisme génétique est exclu dans les phénomènes de duraminisation : on sait qu'il existe de ce point de vue des différences très importantes entre espèces, et, par ailleurs, Nicholds (1965) a calculé une héritabilité au sens large de 0,37 pour le pourcentage de bois de cœur du pin radiata; les deux raisons évoquées ci-dessus au sujet des largeurs de cernes peuvent expliquer, au moins pour partie, cette absence de différence au niveau infra-spécifique.

Les imperfections d'un dispositif comportant deux lignes ( 1 et 8 ) formant lisières sont manifestement responsables des valeurs élevées des « $F$ » ligne, mais une contrepartie bénéfique en résulte : une plus grande variabilité pour l'étude des liaisons vigueur-duraminisation.

Les résultats des tests de comparaison de moyennes sont donnés ci-dessous pour les différentes variables (les lignes soulignées par un même trait ne diffèrent pas significativement entre elles au seuil de 5 p. 100) :

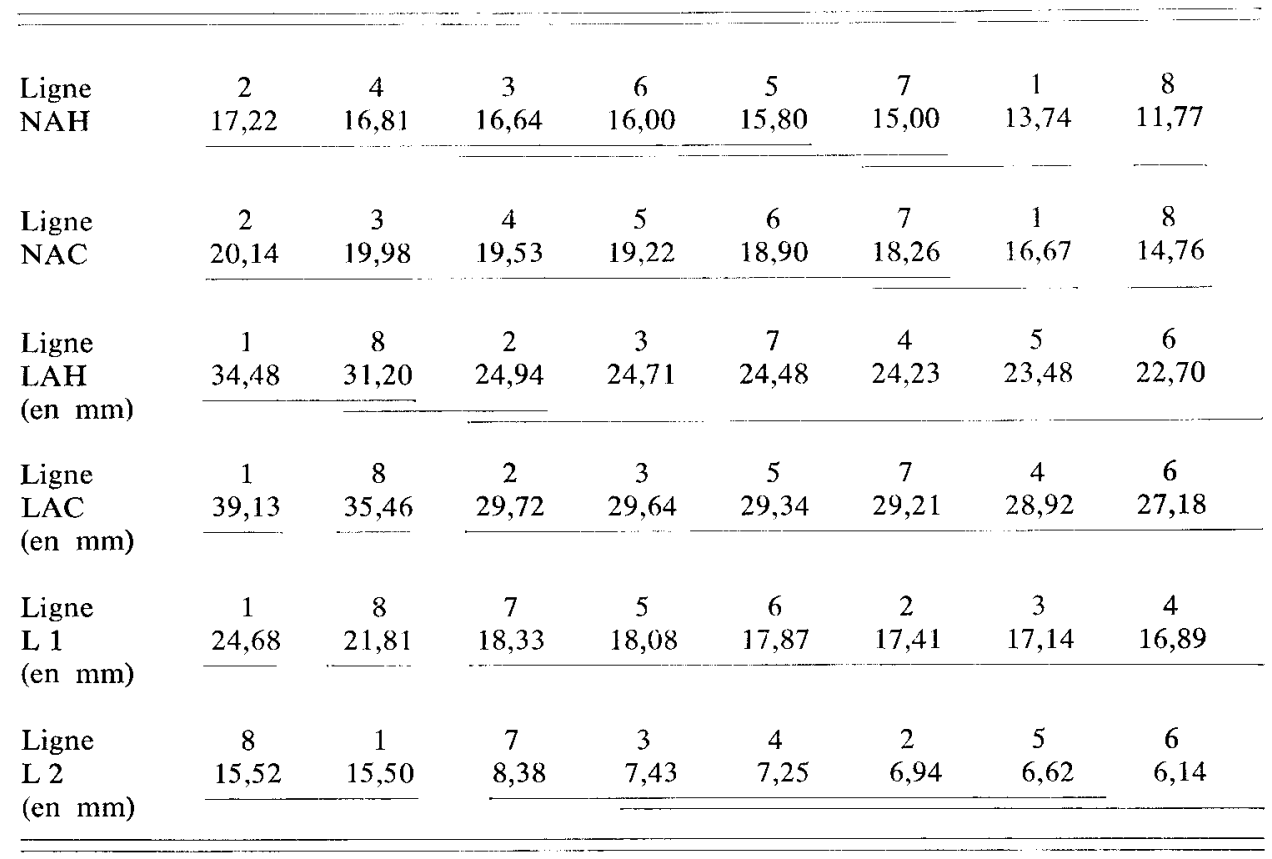


Si l'on examine dabord ces deux derniers résultats relatifs aux largeurs de cernes, on observe une influence évidente des lisières; paradoxalement la future lisière irriguce qui avait une meilleure croissance pour la période 1972-1975, vraisemblablement (GARBAYE, communication personnelle) en raison de conditions édaphiques légèrement plus favorables, perd sa supériorité par rapport à l'autre pour la période 1976-1978 où elle a bénéficié d'un apport d'eau supplémentaire; il y a là encore un effet probable de la compétition, la concurrence entre arbres dans la ligne 1, plus vigoureuse au départ, s`y manifestant déjà nettement durant les 3 dernières années, à un moment où elle ne se faisait encore guère sentir dans la ligne 8 . Pour le reste, on doit seulement noter une amélioration du classement relatif des 3 lignes intérieures irriguées après l'application du traitement, et également une réduction des accroissements annuels entre périodes beaucoup plus forte dans les lignes 2 à 7 , où elle dépasse nettement $50 \mathrm{p}$. 100, que dans les lisières où elle n'est que de 35 p. 100 environ.

Pour les critères de duraminisation, on retrouve le décalage déjà signalé entre la limite «humidité » et la limite «couleur», ce qui n'exclut pas une grande similitude dans le classement des lignes, aussi bien pour les nombres que pour les largeurs. Les deux lisières ont un aubier plus large, mais représenté par un plus petit nombre d'années (nombres-codes NAH et NAC inférieurs). La seule différence significative relative aux lignes intérieures fait apparaître un nombre d'années d'aubier des lignes irriguées 2 et 4 supérieur à celui de la ligne témoin 7 . On doit cependant remarquer que pour 3 critères sur 4 (NAH, NAC et LAH) les résultats ne traduisent pas une symétrie totale par rapport à l'axe du dispositif, comme ce serait le cas si l'effet "lisière » était le seul en cause; on a au contraire toujours $8<1,7<2,6<3$ et $5<4$, ce qui laisse présager un effet possible du traitement.

\subsection{Effets traitement et provenance}

Les valeurs des tests $\ll F »$ sont les suivantes :

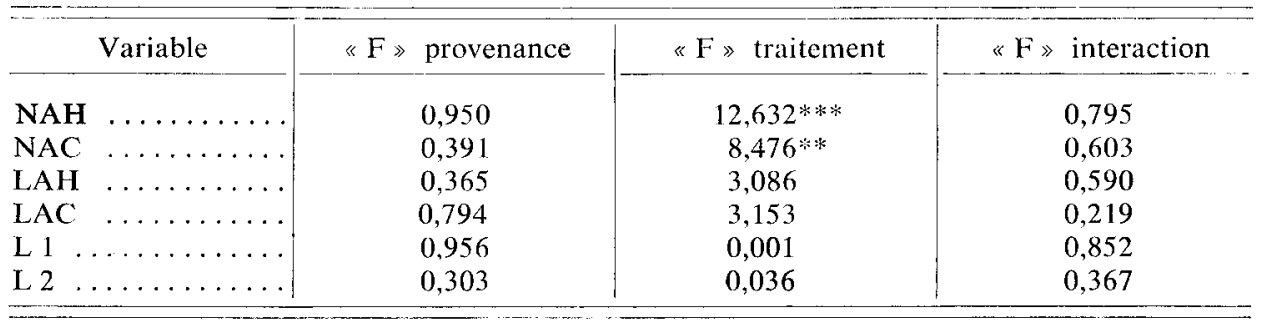

Les valeurs-limites de « $F$ » pour l'effet traitement (1 et 312 D.L.) aux seuils de signification de 5 p. 100,1 p. 100 et 0,1 p. 100 sont respectivement de 3,84 , 6,63 et 10,83 .

Les valeurs moyennes par traitement sont les suivantes (les 4 dernières paires, non significativement différentes, étant données à titre d'information) : 


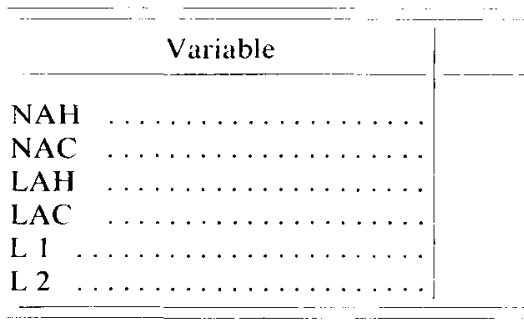

\begin{tabular}{c|c} 
Irrigués & Témoins \\
\hline 16,09 & 14,60 \\
19,07 & 17,77 \\
27,12 & 25,49 \\
31,88 & 30,32 \\
19,05 & 19,04 \\
9.30 & 9,19 \\
\hline
\end{tabular}

Par les deux méthodes, laubier occupe un nombre de cernes significativement plus élevé chez les arbres irrigués que chez les témoins, et a en outre tendance à y être plus large. Ainsi, les effets de la compétition et de la sécheresse apparaissent comme opposés sur le nombre d'années d'aubier, mais, au contraire, assez comparables si l'on se réfère à sa largeur.

Lidentité presque absolue des largeurs de cernes entre arbres traités et témoins mérite d'être soulignée. Dans le même dispositif, Aussenac (1980, op. cit.) avait déjà montré que la sécheresse, après avoir fortement réduit la pousse en hauteur en 1976, n'avait pratiquement plus eu d'effet sur celle de 1977, et Chantal CoyDON (1980) a mis en évidence une compensation entre les largeurs de cernes, significativement supérieures chez les arbres irrigués en 1976 et chez les témoins en 1977 et 1978 .

\subsection{Liaisons entre variables}

Le tableau ci-dessous donne, dans l'ordre, pour chaque couple de variables les coefficients de corrélation entre moyennes de lignes, entre moyennes de provenances, puis le coefficient global pour les 334 arbres de l'expérience, tous multipliés par 1000 :

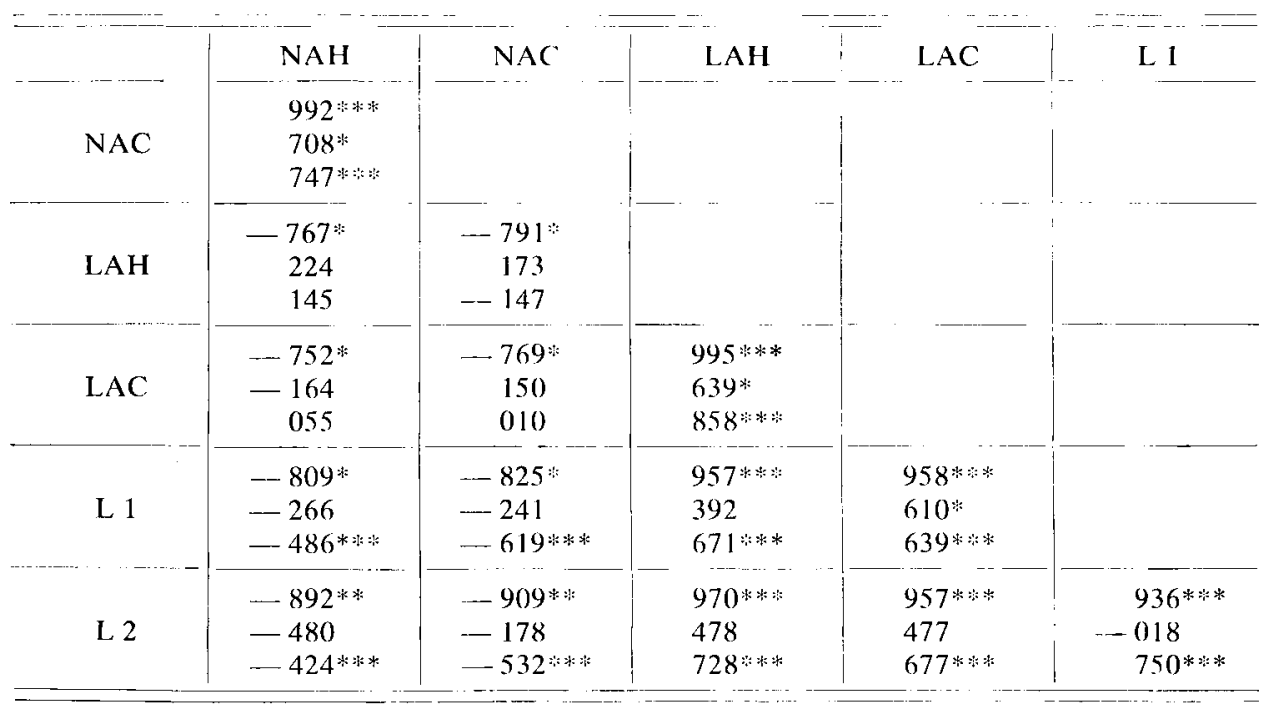


Quel que soit le niveau considéré, les liaisons entre les deux méthodes utilisées pour estimer une même caractéristique (nombre d'années ou largeur de l'aubier) sont significatives. En revanche, l'indépendance entre nombres et largeurs pour deux niveaux sur trois surprend; or le phénomène apparaît comme tout à fait général si l'on considère que la liaison entre moyennes de lignes n'est due qu'aux deux lisières qui donnent des valeurs très supérieures aux autres lignes pour les largeurs d'aubier, mais très inférieures au contraire pour les nombres d'années, et devient elle aussi non significative si on les élimine (voir figure 3). En fait si lon retrouve souvent chez les dominants la même tendance que dans les lisières (aubier large mais n'intéressant qu'un petit nombre d'années), on observe fréquemment parmi les nombreux dominés de ce dispositif un schéma inverse, c'est-à-dire des variations concomitantes de la largeur de la partie vivante de l'arbre et du nombre d'années qu’elle représente, les arbres fortement concurrencés ayant des cernes tellement fins que leur aubier, malgré sa minceur, s’étend sur une période de temps relativement longue. A titre d'exemple, et pour vérifier le bien-fondé de cette explication, le coefficient de corrélation entre NAH et LAH a été calculé pour l'ensemble des dominés : il s'élève à 0,568 , significatif au seuil de 1 p. 1000 (voir figure 4).

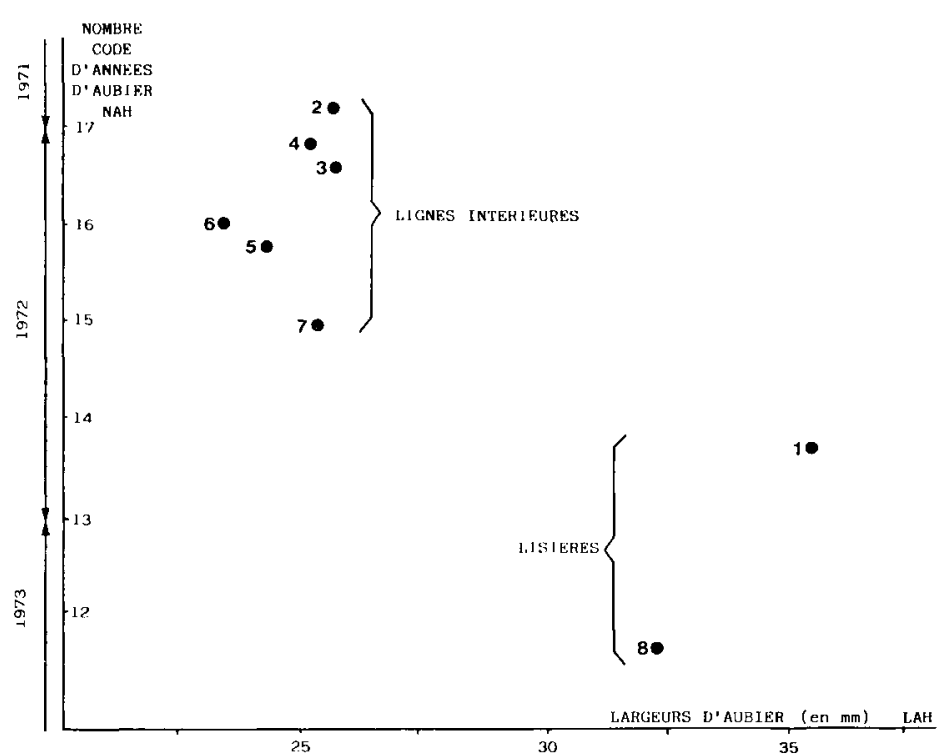

FIG. 3

Corrélation au niveau des moyennes de lignes entre nombre-code d'années et largeur de l'aubier délimité par la teneur en eau.

Correlation between the annual code number and the width of sapwood delimitated according to its moisture content at the line level.

Lignes intérieures : inner lines.

Lisières : border lines. 


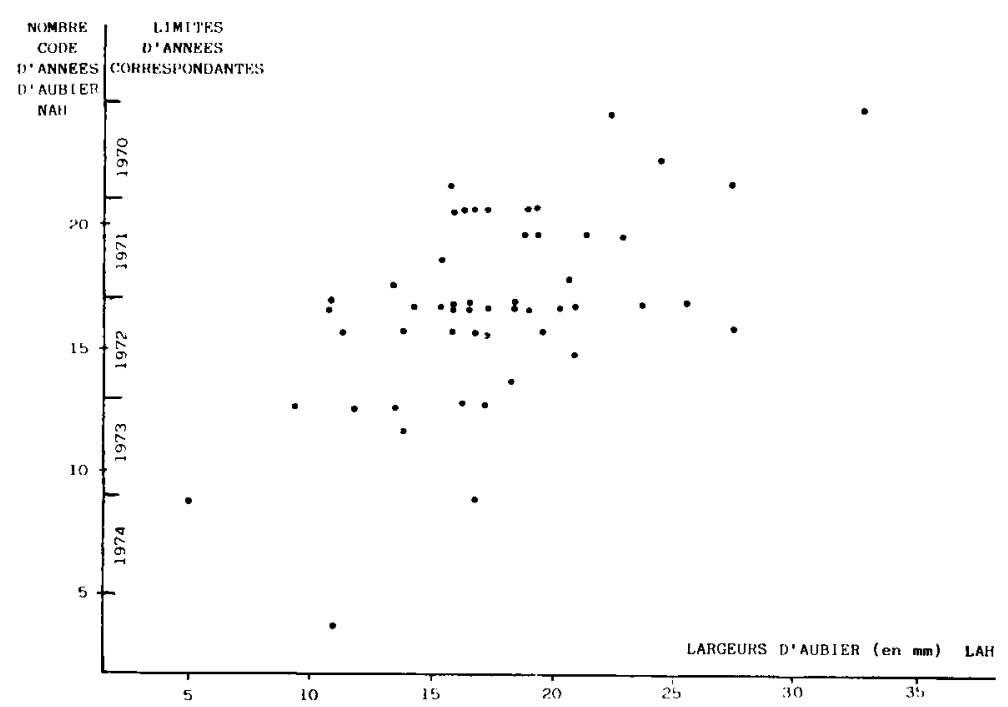

FIG. 4

Corrélation au niveau individuel (arbres dominés seulement) entre nombre-code d'années et largeur de l'aubier délimité par la teneur én eau.

Correlation at the individual level (suppressed trees only) between the annual code number and the width of sapwood delimitated according to its moisture content.

Nombre-code d'années d'aubier : annual code number of sapwood.

Limites d'années correspondantes : corresponding actual limit of years.

Largeurs d'aubier : sapwood width.

En ce qui concerne les critères de vigueur, on note, aussi bien au niveau des moyennes de lignes qu'au niveau intra global, qu'une augmentation de largeur des cernes entraîne la formation d'un aubier plus large, mais s'étendant sur un plus petit nombre d'années.

Au niveau inter-provenances, un effet de la concurrence est probable; c'est en tous cas le seul qui puisse expliquer l'absence totale de liaison entre largeurs de cernes des tranches 1972-1975 et 1976-1978 : certaines provenances vigoureuses ont, faute d'avoir été éclaircies, déjà fortement souffert de la compétition entre tiges ces 3 dernières années, alors que d’autres, à croissance initiale plus lente, ne subissaient pas encore cette influence : ainsi, la provenance Campbell River, qui a eu les plus forts accroissements pour la période 1976-1978, étant avant-dernière en 1972-1975, et, inversement, la provenance Timber, première en 1972-1975, n'était plus que 6" pour la dernière période de mesures.

\subsection{Observations relatives aux zones sèches à l'intérieur de l'aubier}

L'existence inattendue de ces zones, le caractère très variable de leur localisation. les différences qu'elles peuvent présenter suivant les espèces, les individus et les années 
ont déjà été mentionnés (Polge, 1966, op. cit., pp. 132 à 143). Le présent travail a été l'occasion de vérifier le phénomène et d'obtenir certaines indications nouvelles à son sujet.

Tout d'abord, si l'on retrouve dans ce dispositif tous les cas de figure signalés pour le douglas, et notamment la présence de bandes très minces extrêmement pauvres en eau dans le bois final, ou encore l'alternance de plusieurs zones sèches et humides successives, la modalité de loin la plus fréquente correspond à de vastes plages à faible humidité s'étendant sur presque toute la largeur du bois initial.

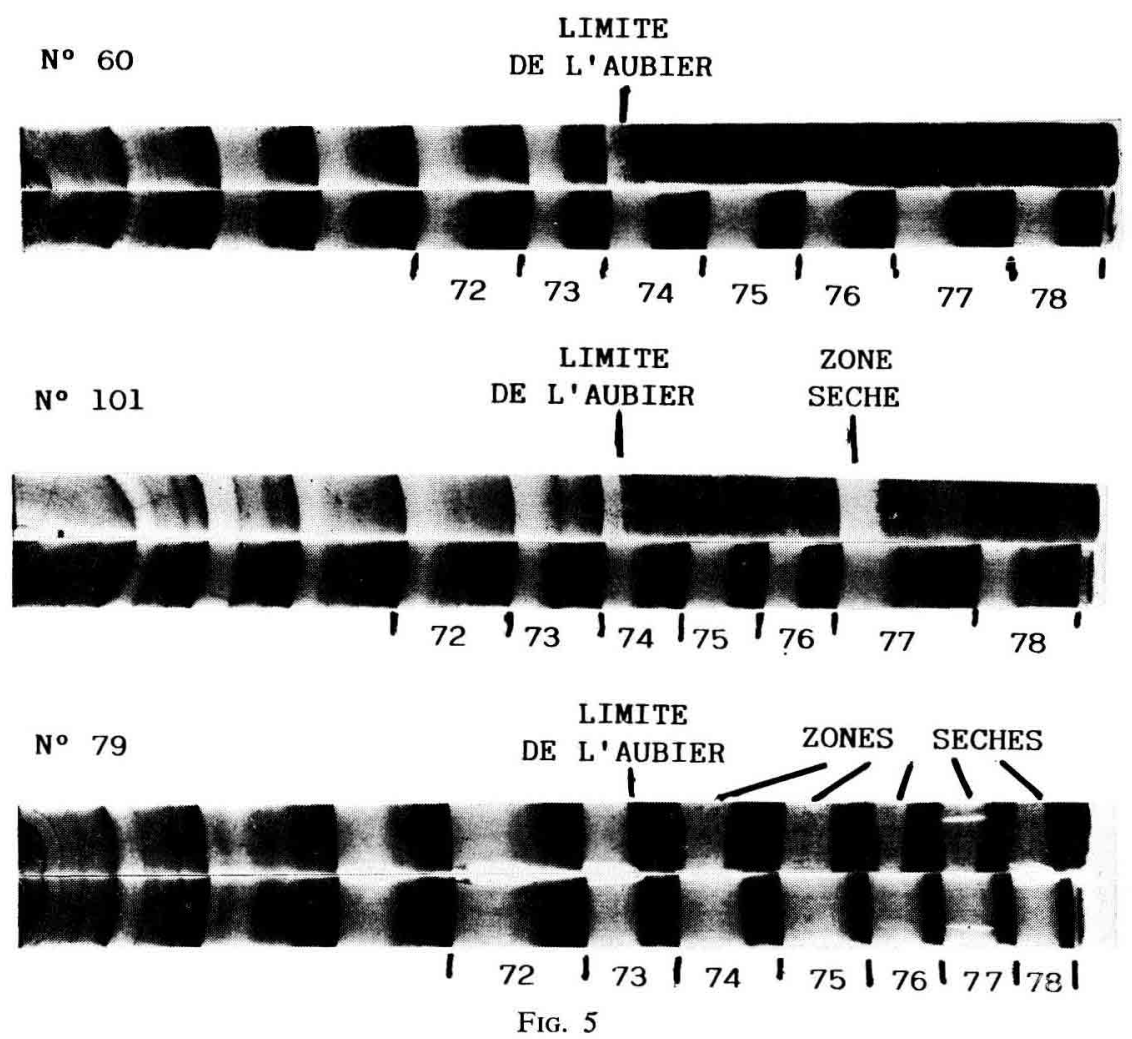

Positifs de radiographies au grossissement 2 d'échantillons à l'état frais et à l'état sec. Positive prints magnified two times of $5 \mathrm{~mm}$ cores $X$-ray images in wet and dry conditions.

Limite de l'aubier : Sapwood limit.

Zone sèche : dry area.

Sur la figure 5 sont regroupés, à titre d'exemple, les positifs de radiographies à l'état frais et à l'état sec, successivement - d'un arbre à aubier «normal » sans zone sèche, sur toute l'étendue duquel on observe peu de variations de teneur en eau, si ce n'est une baisse normale dans le bois final liée à la faible taille de ses cavités cellulaires (il faut cependant tenir compte du fait que, pour avoir le maximum de 
détails dans l'image de la radio à l'état frais, le temps d'exposition a été réduit lors du tirage, en sorte que son niveau de gris n'est pas comparable à celui de son homologue à l'état sec) :

-- d'un arbre à zone sèche unique,

- d'un arbre où toutes les années daubier comportent de larges bandes de bois initial à très faible teneur en eau, dont le cas est particulièrement intéressant car il s'agit d'un arbre à croissance vigoureuse, irrigué en 1976 et 1977, et dont cependant même le dernier cerne, où le flux de sève est le plus intense, est en grande partie sec.

Il existe également des zones à humidité intermédiaire, auquel cas l'eau présente n’est pas disséminée dans l'ensemble des cellules, mais concentrée dans des groupes de fibres eux-mêmes dispersés dans toute la partie d'accroissement concernée (figure 6) et dont l'étude anatomique n'a pas fait apparaître de différences par rapport à leur voisinage.

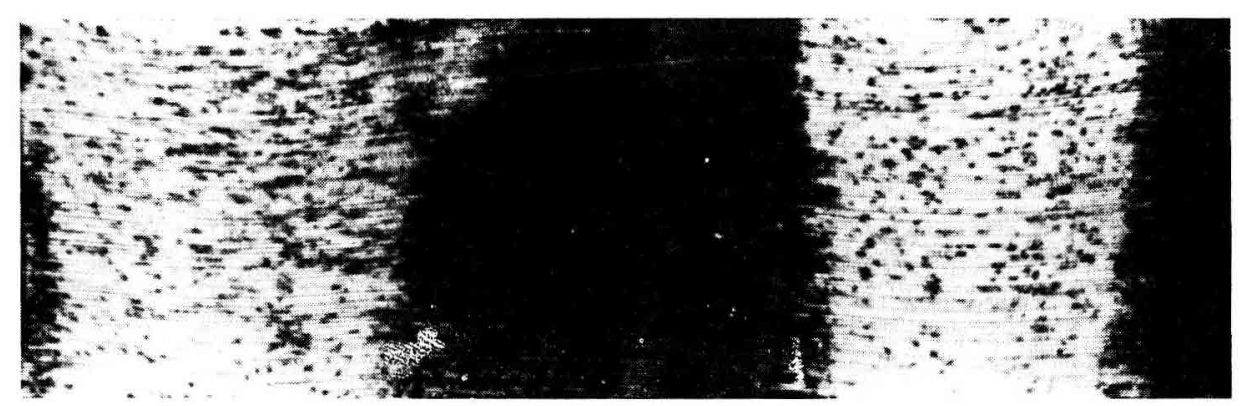

FIG. 6

Répartition de l'eau dans des zones à humidité réduite du bois initial (positif de radiographie au grossisement 10).

Water distribution in relatively dry early wood (positive print of X-ray image magnified 10 times).

Un inventaire complet des zones sèches ayant au moins $0,5 \mathrm{~mm}$ de large en grandeur réelle dans le sens radial a donné les résultats suivants (les numéros des provenances correspondent à leur ordre de succession de gauche à droite sur la figure 1) :

\begin{tabular}{|c|c|c|c|c|c|c|c|c|c|c|c|}
\hline Provenances $\ldots .$. & 1 & 2 & 3 & 4 & 5 & 6 & 7 & 8 & 9 & 10 & 11 \\
\hline $\begin{array}{c}\text { Nombre darbres à zones } \\
\text { sèches } \ldots \ldots \ldots \ldots \ldots\end{array}$ & 9 & 13 & 14 & 13 & 17 & 5 & 17 & 14 & 10 & 7 & 4 \\
\hline $\begin{array}{c}\text { Nombre d'années à zones } \\
\text { sèches } \ldots \ldots \ldots \ldots \ldots\end{array}$ & 5 & 6 & 6 & 8 & 5 & 5 & 6 & 7 & 4 & 6 & 2 \\
\hline $\begin{array}{c}\text { Nombre de cernes à zones } \\
\text { sc̀clies } \ldots \ldots \ldots \ldots \ldots\end{array}$ & 21 & 34 & 21 & 30 & 25 & 7 & 24 & 29 & 20 & 11 & 4 \\
\hline
\end{tabular}


Quel que soit le critère retenu, la variabilité entre provenances est importante, mais leur classement relatif diffère, certaines ayant un plus petit nombre d'arbres concernés, mais, pour chacun d’eux plus d’années à zones sèches, et inversement.

Les provenances 11 (Granite Falls) et 6 (Marion Creek), dont l'aubier apparaît comme le plus «normal» du point de vue de la répartition de l'eau, ont des aires d'origine très différentes aussi bien en altitude qu'en latitude; Marion Creek a le bois initial le plus dense des 11 provenances, mais Granite Falls est tout à fait dans la moyenne; sans doute convient-il d'observer que la première est en lisière du dispositif, au contact d'un peuplement de hêtres qui a pu l'abriter des vents dominants d’Ouest, et réduire ainsi son évapo-transpiration.

Une étude de la répartition par années, toutes provenances confondues, des arbres à zones sèches n'est possible que si l'on exclut 1972 et 1973 pour lesquelles la duraminisation est déjà intervenue chez de nombreux individus. Une liaison apparaît alors avec les densités minimales annuelles mesurées par Chantal Coydon (1980, op. cit.); les valeurs moyennes sont les suivantes :

\begin{tabular}{r|r|r|r|r|r}
\hline \hline Années & 1974 & 1975 & 1976 & 1977 & 1978 \\
\hline Densités minimales annuelles ........ & 246,7 & 245,5 & 254,1 & 253,6 & 261.8 \\
Pourcentages d'arbres à zones sèches & $11,4 \%$ & $23,7 \%$ & $7,2 \%$ & $3,9 \%$ & $0.9 \%$ \\
\hline
\end{tabular}

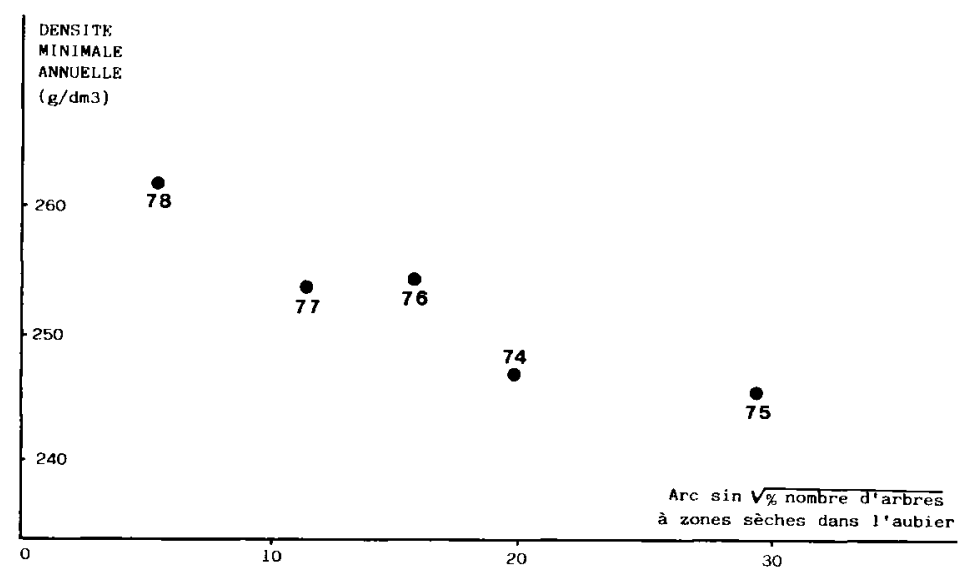

FIG. 7

Corrélation entre la densité minimale annuelle et la fréquence annuelle des arbres à zones sèches dans l'aubier.

Correlation between minimum annual density and the annual frequency of trees with dry areas in the sapwood. 
Après transformation en Arc sinus racine carrée des pourcentages qui sont très variables, mais dans l'ensemble assez faibles, le coefficient de corrélation entre les deux séries de données est de $-0,93$ significatif au seuil de 5 p. 100 pour un nombre de degrés de liberté réduit à 3 (figure 7); ainsi, les années où la densité du bois initial est la plus faible ont une plus forte probabilité de présence de zones sèches dans ce même bois initial. Cela n'est pas tout à fait surprenant, car la formule de la perméabilité du bois fait intervenir, à la puissance 4 , le diamètre de l'orifice des ponctuations aréolées; on sait que celui-ci est plus grand pour les trachéïdes du bois initial que pour celles du bois final, et, par analogie, on peut envisager qu'il augmente avec le diamètre et diminue avec l'épaisseur de la membrane des cellules du premier, ce qui rendrait le départ de l'eau d'autant plus aisé que la densité du bois initial serait plus faible. Il n'existe malheureusement dans la littérature pas de renseignements précis sur les variations entre individus de ces caractéristiques anatomiques pour appuyer cette hypothèse, et, par ailleurs, toutes les tentatives pour lier la présence des zones sèches de l'aubier à des facteurs du climat sont demeurées vaines.

\section{Discussion et conclusion}

A la différence des diverses études publiées jusqu'ici sur l'étendue de laubier dans les arbres forestiers, celle dont il est rendu compte ci-dessus est basée sur deux critères macroscopiques simples de délimitation (différence de teneur en eau et changement de couleur), et, pour chacun d'eux, sur deux modes d'expression qui apparaissent au terme de ces observations comme beaucoup plus indépendants qu'on ne l'aurait pensé, et dont l'un se réfère à la très traditionnelle largeur de l'aubier, et l'autre, au nombre d'années sur lequel il s'étend. En outre, grâce à la méthodologie utilisée, les mesures ont pu être faites sur un très large échantillonnage, ce qui a permis, en dépit des insuffisances du dispositif, de procéder à une analyse statistique des résultats.

Les deux méthodes concordent pour montrer que la coloration du bois de cœur intervient nettement après la diminution de la teneur en eau, et le fait que le décalage entre les deux phénomènes varie de façon importante d'un individu à l'autre amène à penser qu'ils résultent de processus physiologiques qui ne sont pas liés entre eux de façon automatique et fonctionnelle.

Les liaisons positives étroites trouvées entre critères de vigueur et largeur de l'aubier. tout comme les différences relevées entre lisières et lignes intérieures, concordent bien avec les données anciennes de la littérature (SмIтH et al., 1966) et aussi avec des résultats récents de Granier (1981) qui, pour la même essence et dans la même forêt, a mis en évidence une relation linéaire très nette entre masse foliaire et surface d'aubier.

Les corrélations négatives entre accroissements annuels et nombres d'années d'aubier étaient au contraire inattendues et montrent l'intérêt de ce nouveau mode d'expression de l'importance de l'aubier; il devrait, semble-t-il, être désormais systématiquement utilisé, concurremment avec d'autres (largeur, surface, pourcentage...) chaque fois que l'on cherche à connaître les effets d'un des facteurs de la production forestière (sylviculture, sol, hérédité) sur le développement de l'aubier : 
il est clair en effet que, si les utilisateurs ne sont concernés que par la quantité d'aubier qu'ils trouvent dans leurs grumes, les propriétaires forestiers devraient également se soucier du nombre d'accroissements annuels concernés, et, lorsque par exemple la présence d'un aubier important correspond à un défaut, préférer les génotypes ou les traitements sylvicoles ayant pour effet, toutes choses égales par ailleurs, de minimiser le nombre d'années de production perdues ou dévalorisées de ce fait.

Enfin, le fait qu'arbres de lisières et arbres irrigués aient les uns et les autres un aubier plus large est intéressant du point de vue de la biologie des espèces ligneuses: pour les premiers il peut s'agir d'une adaptation à une transpiration supérieure liée à l'ampleur de leur masse foliaire, et ceci concorde bien avec l'interprétation « finaliste » de la duraminisation proposée par BAMBER (1970, op. cit.), mais l'explication n'est pas valable pour les seconds pour lesquels il y a augmentation, non pas des besoins en eau (il n'y a pas de différence de croissance entre arbres traités et témoins), mais de la disponibilité. La contradiction n'est peut-être qu'apparente, car, face au très fort déficit hydrique de 1976, le flux transpiratoire des témoins a pu être réduit, soit du fait de la chute importante d'aiguilles dont ils ont souffert (AuSSENAC, communication personnelle), soit du fait d'un autre mécanisme régulateur (fermeture des stomates notamment) entraînant indirectement, et par voie de conséquence, la mise en route de celui qui, d'après BAMBER, conditionne la formation du bois de ccur, ce qui, naturellement n'aurait pas été le cas pour les arbres irrigués.

Resu pour publicution en octobre 1981.

\section{Remerciements}

Que soient vivement remerciés ici :

- $\mathrm{M}^{\mathrm{me}}$ Gigout, qui a procédé au traitement des données sur ordinateur,

- M. Houssement, qui a réalisé l'ensemble des radiographies,

- M. Michel, qui a tiré plus de 1350 agrandissements photographiques dans le cadre du présent travail.

\section{Summary \\ Effect of competition and of water availability on the sapwood of douglas fir}

The 334 trees of the experiment are 13 years old Douglas firs of 11 different provenances, of which 50 p. 100 have been irrigated in 1976 and 1977. Two methods have been used to deliminate the sapwood, based one on its higher moisture content (by comparison between X-ray images of wet and dry increment cores), the other on the discoloration at the heartwood boundary observed on the cores in dry conditions. Two modalities of quantification have been used : the width and the number of annual increments of the sapwood.

The change of colour occurs almost one year after the decrease of moisture content, and the corresponding gap is about $5 \mathrm{~mm}$ in radial direction.

There is no influence of the provenance, but a very significant one of the line within the experimental plot, due to two borders in which the sapwood is larger, but lies on 
fewer annual increments. The effect of the treatment is very significant on the number of sapwood rings, that is higher on the irrigated trees, in which the sapwood is also wider.

The correlations between both methods are very narrow, but there is no significant relationship between the modalities of quantification, because the number of sapwood rings and its width varies in opposite direction amongst the dominants and in the same direction amongst the suppressed trees.

Many dry areas have been numbered in the sapwood; mostly, they are larger than $0.5 \mathrm{~mm}$ and are located within the early wood. It is difficult to relate them to climatic factors, but there is a strong negative correlation between their relative frequency and the minimum wood density of the annual ring.

\section{Références bibliographiques}

Aussenac G., 1980. Premiers résultats d'une étude de l'influence de l'alimentation en eau sur la croissance des arbres dans un peuplement de douglas [Pseudotsuga menziesii (Mirb.) Franco]. Rev. for. fr., 2, 167-172.

Bamber R.K., Humphreys F.R., 1965. Variations in sapwood starch level in some Australian forest species. Aust. For., 29, 15-23.

BAmber R.K., 1970. The histochemistry and anatomy of parenchymatous tissues of the wood of some conifers and the relationship to permeability. M.Sc. Thesis, Macquarie university.

Bamber R.K., 1972. Properties of the cell walls of the resin canal tissue of the sapwood and heartwood of Pintus lambertiana and Pints radiata. J. Inst. Wood Sci., 6 (1), 32-35.

Bamber R.K., 1975. Comparative studies of sapwood and heartwood. Ph.D. Thesis, Macquarie University.

Chattaway Maragaret, 1952. The sapwood-heartwood transition. Aust. For., 16. 25-34.

CoYdon Chantal, 1980. Influences de certains facteurs climatiques sur l'accroissement et la structure du bois de jetures douglas [Pseudotsuga menziesi (Mirb.) Francol. D.E.A.. Université de Nancy.

Frey-WYssi.ing A., Bosshard H.H., 1959. Cytology of the ray cells in sapwood and heartwood. Holzforschung, 13, 129-137.

Granier A. (1981), Etude des relations entre la section du bois d'aubier et la masse foliaire chez le douglas [Pseudodouglas menziesii (Mirb.) Franco], Ann. Sci. for., 38, 4, 503-512.

Nicholls J.W.P., 1965. Heritability of heartwood formation in Pinus radiata (D. Don.). Nature, 207, 320.

Polge H., 1964. Délimitation de l'aubier et du bois de cour par analyse densitométrique de clichés radiographiques. Ann. Sci. for., 21, 4, 605-623.

POLge H., 1966. Etablissement des courbes de variation de la densité du bois par exploration densitométrique de radiographies d'échantillons prélevés à la tarière sur des arbres vivants. A pplications dans les domaines technologique et physiologique. Thèse Doctorat d'Etat, Université de Nancy.

Priestley J.H., 1932. The growing tree. Forestry, 6, 105-112.

Rudman P., 1966. Heartwood formation in trees. Nature, 210, 242-257.

SMirh J.H.G., Walters J., Wellwood R.W., 1966. Variations in sapwood thickness of douglas-fir in relation to tree and section characteristics. For. Sci., 1, 97-103.

Stewart C.M., 1967. Moisture content of living trees. Nature, 214, 138-140. 